\title{
Investissements ou accaparements fonciers en Afrique ? Les visions des paysans et de la société civile au Mali
}

Investments or land grabbing in Africa? Farmers and civil society's views in Mali

Amandine Adamczewski, Jean-Yves Jamin, Benoît Lallau et JeanPhilippe Tonneau

\section{(2) OpenEdition}

Édition électronique

URL : http://journals.openedition.org/developpementdurable/9424

DOI : 10.4000/developpementdurable.9424

ISSN : 1772-9971

Éditeur

Association DD\&T

Référence électronique

Amandine Adamczewski, Jean-Yves Jamin, Benoît Lallau et Jean-Philippe Tonneau, «Investissements ou accaparements fonciers en Afrique ? Les visions des paysans et de la société civile au Mali »,

Développement durable et territoires [En ligne], Vol. 3, n 3 | Décembre 2012, mis en ligne le 09 décembre 2012, consulté le 01 mai 2019. URL : http://journals.openedition.org/developpementdurable/9424 ;

DOI : 10.4000/developpementdurable.9424

Ce document a été généré automatiquement le 1 mai 2019.

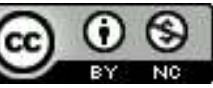

Développement Durable et Territoires est mis à disposition selon les termes de la licence Creative Commons Attribution - Pas d'Utilisation Commerciale 4.0 International. 


\section{Investissements ou accaparements fonciers en Afrique? Les visions des paysans et de la société civile au Mali}

Investments or land grabbing in Africa? Farmers and civil society's views in Mali

Amandine Adamczewski, Jean-Yves Jamin, Benoît Lallau et JeanPhilippe Tonneau

1 Depuis la crise alimentaire de 2008, le nombre et l'ampleur des projets d'investissements agricoles à grande échelle (plus de mille hectares) ont fortement augmenté (Von Braun et Meinzen-Dick, 2009). Ils se traduisent par des achats de terre ou par des concessions de baux de longue durée (Cotula et al., 2009; Zoomers, 2010). Ces acquisitions foncières sont principalement concentrées dans les pays du Sud, et plus particulièrement en Afrique subsaharienne (Chouquer, 2011). En 2009, à partir de données obtenues auprès de l'ONG GRAIN, la Banque mondiale a publié un rapport sur les transactions foncières ${ }^{1}$. Selon ce rapport, 45 millions d'hectares auraient été concernés en 2009, environ dix fois plus que la moyenne des années précédentes. En 2011, le Land Matrix ${ }^{2}$ avance le chiffre de 203 millions d'hectares pour la période 2000-2010, dont 71 millions ayant fait l'objet de contrats signés.

2 Cette ruée - mondiale - sur les terres est inédite, tant par son ampleur que par ses finalités. Il ne s'agit plus seulement pour des firmes privées d'assurer leurs approvisionnements en matières premières industrielles (plantations d'hévéas de Michelin au Brésil ou de Firestone au Libéria, de palmiers à huile d'Unilever au Nigéria, etc.) ou de cultiver des fruits pour l'exportation (Dole et Del Monte en Amérique centrale ou au Cameroun, etc.). Il s'agit pour des entreprises mais, aussi, pour des États en manque de terres arables (Libye, Arabie Saoudite, Chine, Inde, USA, pays européens) d'implanter dans d'autres pays des cultures alimentaires et des cultures énergétiques, pour garantir leur sécurité alimentaire et pallier la raréfaction des énergies fossiles (Burnod et al., 2011). L'Afrique est le continent privilégié de ces nouveaux investissements (Anseeuw et 
al., 2012). Elle est généralement décrite comme disposant d'importantes réserves foncières peu ou pas valorisées. La Banque mondiale qualifie ainsi l'Afrique de "Géant endormi » n'attendant que les investisseurs pour s'éveiller à la croissance agricole (World Bank, 2009). Cette vision est discutée par ceux qui dénoncent « des accaparements fonciers » (Cotula et al., 2009; Alternatives Économiques, 2009; Baxter, 2010). Pour GRAIN, les détenteurs de capitaux financiers « arrachent » des terres productives à des populations ou à des États faibles (weak states), aux capacités d'actions (financières, politiques, sociales) limitées (GRAIN, 2008).

3 Les intérêts des investisseurs, publics ou privés, sont évidents : d'une part, garantir leur approvisionnement en aliments, en matières premières et en énergie; d'autre part, développer leurs profits (Olivier Martin, 2011; Cicodev Africa, 2011 ; Le Quorum, 2011). Les États hôtes justifient ces projets comme porteurs de développement, par l'apport de capitaux, la création de valeur et les emplois qu'ils engendrent. Ils soulignent la contribution à deux objectifs politiques majeurs : moderniser une agriculture considérée comme " archaïque », et développer le potentiel agricole à travers la mise en valeur de terres considérées comme peu productives.

4 Les modèles techniques promus sont intensifs: variétés à haut rendement, parfois obtenues par modifications génétiques, grande mécanisation, forte utilisation des produits phytosanitaires, et, fréquemment, irrigation. Le coût environnemental de ces modèles est souvent élevé. Mais la principale critique porte sur les mécanismes de spoliation de terres que ces projets motivent: la société civile souligne que ces projets découlent d'abord d'une course à la terre et de la constitution d'une rente foncière. Plusieurs auteurs avancent les termes d'accaparement de terres et d'appropriation foncière (Rochegude, 2011 ; Teyssier et al., 2010 ).

5 Les conséquences de ces grands projets pour les populations locales peuvent être l'expulsion, la diminution des ressources disponibles (bois de feux, pâturages,...) ou la réduction des terres cultivables. Les terres concernées sont rarement vierges d'activités humaines, et les droits des populations en place sont peu pris en compte (Anseeuw et al., 2012). La situation des paysans qui exploitent ces terres (agriculture pluviale, élevage extensif, chasse, cueillette...) est rendue fragile par le flou des lois qui reconnaissent peu les droits fonciers coutumiers. La substitution des paysans par les projets est niée ou justifiée par les impératifs du développement et la nécessité du passage d'une agriculture archaïque à une agriculture moderne (World Bank, 2008). Les coûts sociaux sont alors présentés comme le prix à payer pour que la nation accède à un «avenir meilleur ». Ces positions ont fait l'objet de nombreuses critiques (Mazoyer et al., 2008; Delcourt, 2010 ; Dufumier, 2012). En 2008, le directeur de la FAO a qualifié certains projets d'investissement de "forme de néo-colonialisme $e^{3}$ ».

6 Ces projets induisent-ils réellement du développement ? La faiblesse des impacts macroéconomiques a été soulignée par la Banque mondiale en 2010. Certains projets entraînent une destruction nette d'emplois liée au remplacement d'agricultures familiales intensives en travail par des agricultures intensives en capital, fondées sur l'économie du facteur travail ; certains investisseurs (comme la Chine) ont même recours à une main-d'œuvre importée du pays d'origine (Lafargue, 2005). La Banque mondiale a calculé, sur quatorze pays étudiés, une moyenne de 0,005 emploi à l'hectare accessible aux populations locales (World Bank, 2010, 45). On note, également, la faiblesse des recettes fiscales induites, puisque les investisseurs bénéficient de multiples exonérations, et payent, au final, souvent moins de taxes que les exploitations familiales. 
7 Enfin, les investissements ne contribuent que peu à l'autosuffisance alimentaire du pays d'accueil, puisqu'ils ont, par nature, une vocation exportatrice. La contribution à la sécurité alimentaire des pays importateurs est également souvent plus faible qu'annoncée, du fait, en particulier, de la part croissante des superficies destinées à des utilisations non alimentaires, $24 \%$ en 2011 selon la Land Matrix Database (Anseeuw et al., 2012)

8 Pour répondre à ces critiques, les acteurs institutionnels, en premier lieu la Banque mondiale, promeuvent les principes de l'agro-investissement responsable (Responsible Agricultural Investment - RAI, World Bank, 2010) qui ont pour objectifs de garantir de « réels » investissements et d'éviter la spéculation foncière. De nombreux appels ont été lancés en ce sens (De Schutter, 2009). Est également mise en avant la complémentarité avec une agriculture familiale dont la Banque mondiale (Deininger \& Binswanger, 1999), reconnaît les avantages en termes d'efficacité, d'équité et de lutte contre la pauvretét. La solution préconisée passerait par une meilleure articulation, "gagnant-gagnant " (Lautier, 2002), entre les agricultures familiales et agro-industrielles. Cependant, les principes de RAI sont basés sur des démarches volontaires des investisseurs; ils n'ont aucun caractère contraignant et sont de fait peu appliqués. On peut s'interroger sur la portée d'appels à la «bonne volonté " lorsqu'il est question de sécurité nationale, alimentaire et énergétique ${ }^{5}$. Le principal effet de ces principes « responsables» semble être d'apporter une caution et une respectabilité, aux modèles de développement basés sur les investissements étrangers et le secteur privé.

9 Face au poids des gouvernements, des investisseurs et des bailleurs de fonds comme la Banque mondiale, quelles peuvent être les réactions des sociétés civiles africaines? Celles-ci s'organisent à trois échelles: celle des mobilisations locales; celle des organisations de dimension nationale; celle, enfin, des ONG internationales, relayant les revendications locales et nationales dans des médias mondiaux et dans les instances internationales.

10 Dans de nombreux pays du Sud, les institutions internationales, notamment les bailleurs de fonds multilatéraux, appuient l'émergence des acteurs dits " de la société civile ». Ce sont des entreprises privées, coopératives, associations, ONG, considérées comme les éléments indispensables d'une vie politique plus responsable, plus ouverte et plus représentative. L'appel à la société civile traduit une volonté de restituer à la société des pouvoirs économiques, sociaux ou d'expression que les États auraient usurpés. La société civile est alors définie comme l'ensemble des institutions où les individus poursuivent des intérêts communs sans interférence de l'État (Rangeon, 1986). Toutefois, l'unité de ces groupes supposerait qu'un État fort existe, un État aux idéologies marquées, auquel ces groupes s'opposeraient dans une « union sacrée ».

11 Ce n'est pas le cas, au Mali, en zone Office du Niger (ON). La zone est éloignée de Bamako, de ses syndicats et de ses ONG, plus en relation avec leurs homologues internationaux. Au fil du temps, différents groupes s'opposent, ou s'allient, à l'État, à des degrés variables. Ils n'échangent que peu entre eux, au sein de l'ON et avec les groupes de la capitale ou des autres régions. ONG et syndicats communiquent peu et ne revendiquent pas les mêmes droits, ne prônent pas les mêmes idées, entre autres du fait d'un décalage assez fort entre urbains/salariés/fonctionnaires de la capitale et ruraux/paysans/éleveurs des régions. Il n'existe ici ni unité ni continuité dans l'espace et dans le temps au sein de la "société civile ». Nous ferons donc référence aux sociétés civiles au Mali, pour tenir compte de 
cette complexité, comme l'ont fait dans d'autres contextes Khilnani (2001) ou Otayek (2002).

12 Ces sociétés civiles peuvent-elles résister et défendre les paysans et les pasteurs touchés par les projets? Peuvent-elles les aider à s'organiser? Peuvent-elles imaginer des synergies et des complémentarités entre agricultures familiales et agriculture d'entreprise qui permettraient un vrai partenariat « gagnant-gagnant »? Pour apporter des éléments de réponse à ces questions, nous nous appuierons sur une étude de cas, celle du projet Malibya. Nous analyserons la mise en œuvre du projet et les tentatives de réactions de la société civile. Nous essaierons alors d'identifier les possibilités d'action qui s'offrent aux populations confrontées à ces investissements et aux organisations qui les représentent, et leur impact possible.

\section{La mise en œuvre d'un projet agro-industriel au Mali}

13 L'analyse de la mise en œuvre du projet Malibya au Mali, permet de discuter, à partir d'un projet d'investissement emblématique, des motivations de l'investisseur, de celles de l'État d'accueil, le Mali, et des problèmes que pose cet investissement. Nous détaillerons, dans un premier temps, les modalités de mise en œuvre du projet, ainsi que ses implications pour les populations locales. Nous mettrons, ensuite, en évidence les difficultés qu'ont ces populations, malgré des relais au niveau national, et même international, pour peser sur le cours d'un tel projet et en limiter les effets négatifs. 68 enquêtes ont été menées entre 2010 et 2011 auprès de six villages et de deux communes concernés par le projet Malibya. Les responsables du projet (représentant libyen au Mali, ingénieurs locaux), les responsables des entreprises travaillant dans le cadre du projet et les responsables maliens en charge du suivi du projet, ont également été rencontrés. Les données provenant de ces entretiens ont été complétées par l'analyse de documents: convention avec l'État, étude de faisabilité, études d'impact environnemental et social.

\subsection{Le projet Malibya et ses impacts}

\subsubsection{La genèse du projet Malibya}

Souhaitant développer et moderniser son agriculture, mais ne disposant pas des moyens financiers nécessaires, ni en propre, ni à travers ses bailleurs de fonds habituels (Banque mondiale, AFD, KFW...), l'État malien a lancé un appel aux investissements étrangers. Le gouvernement libyen a été l'un des premiers à répondre à cet appel. Le projet Malibya, initié en 2008, porte sur 100000 ha. C'est un partenariat public-public entre l'État malien et l'État libyen à travers The Libya Africa Investment Portfolio (LAP), société pilotée par le directeur de cabinet de Mouammar Kadhafi. Il est mis en œuvre par une société $100 \%$ libyenne, Malibya, qui bénéficie des avantages du code des investissements malien, actualisé en 2009.

Le projet est localisé dans la plus grande zone irriguée d'Afrique de l'Ouest, l'Office du Niger'. Il prévoit le développement de la riziculture intensive irriguée par gravité ou sous pivot, de la culture de la canne à sucre irriguée sous pivot, et de l'élevage en stabulation. Une première tranche de 38 millions d'euros a été mobilisée pour réaliser les premières infrastructures (canal, route, aménagement prévu de $25000 \mathrm{ha}$ ). Une route et un canal 
d'une longueur de 40 kilomètres ont été construits. Les 18 premiers kilomètres du canal sont communs au projet Malibya et à de futurs projets agro-industriels maliens.

Signée en mai 2008, la convention entre les deux parties n'a été rendue publique qu'à la mi-2009. Elle précise les droits, les devoirs et les engagements des États, ainsi que les avantages accordés au projet (indemnisation des populations, tarif bas pour l'eau d'irrigation, accès privilégié à l'eau durant la période d'étiage, ...). Le Mali a offert gratuitement des terres au projet, pour un bail de 50 ans renouvelable. Ces terres sont réputées « libres » d'occupation, à charge pour le gouvernement malien de s'occuper du déplacement des populations - et de leur éventuelle indemnisation.

\subsubsection{Un projet négocié à la marge de la législation en vigueur...}

17 Les textes encadrant les investissements au Mali prévoient qu'un projet doit réaliser, avant toute action de terrain, des études d'impact environnemental et social (EIES), se fondant sur des consultations des populations locales. Ces études doivent permettre d'analyser le fonctionnement humain et environnemental du milieu et d'évaluer les répercussions du projet (Adamczewski et al., 2010). Dans le cadre du projet Malibya, l'étude d'avant-projet pour la construction du canal et l'EIES n'ont été réalisées qu'après le démarrage des travaux, à la fin de 2008. L'entreprise libyenne a débuté le chantier sans avoir obtenu le permis environnemental, accordé par l'État après validation des études EIES. Aucune consultation des populations locales n'a été organisée. Les topographesgéomètres et l'entreprise chinoise, chargée de la réalisation des travaux, se sont installés sans que les populations n'aient été informées de leur arrivée. Des réunions ont eu lieu après les travaux de construction du canal d'irrigation pour exposer aux paysans les règles de passage, d'utilisation... Il ne s'agit pas réellement de consultations des populations au sens de Rowe et Frewer (2004) pour recueillir leurs attentes et discuter des évolutions du projet, mais plutôt de réunions d'information post travaux. Pour la partie libyenne, « tout cela » était du ressort de l'État malien, qui n'a, en fait, pas les moyens de mener ces "mesures d'accompagnement». Les investissements réalisés représentent moins de $3 \%$ de ceux projetés et annoncés dans les études préliminaires. Cette " panne » est bien antérieure à l'éclatement de la guerre civile libyenne. En résumé, la législation n'a pas été respectée et les engagements financiers pris n'ont pas été tenus.

\subsubsection{Les impacts du projet sur les villageois}

La base de vie du projet (où ont été logés les ingénieurs en charge de la construction du canal d'irrigation) a été construite sur un couloir de transhumance du bétail (Brondeau, 2011). La reconnaissance de ce lieu de passage d'animaux n'ayant jamais été officialisée par l'État, les populations locales peuvent difficilement revendiquer le respect de leurs droits. Et le projet peut renvoyer la responsabilité de cette erreur sur l'État. La construction du canal d'irrigation a entrainé le déplacement de certains villages et l'abandon des jardins maraîchers établis en bordure du fala (bras mort du fleuve). Les déblais issus du creusement du canal ont été déversés dans les champs voisins, en empêchant désormais l'exploitation en mil pluvial, puisqu'il s'agit de terre stérile remontée du sous-sol, et, de surcroît, déposée sous forme de tas qui n'ont même pas été aplanis.

Le canal a divisé en deux des villages, ou séparé des villages de leurs hameaux ou de leurs champs. Sur une longueur de plus de $40 \mathrm{~km}$, seuls trois ponts permettent de traverser le 
canal et donc de relier les deux rives. Le tracé de ce canal a fait l'objet de nombreuses négociations entre la Libye et l'État malien. Un premier tracé avait été décidé par la Libye en 2008, sans consultation de la partie malienne. L'étude faisait alors état de 16 villages à déplacer, d'infrastructures de santé et d'écoles à développer, etc., pour un coût global de 24 millions d'euros. Comme la convention prévoit que l'État malien livre au projet libyen des terres libres de toute occupation, la Libye lui a demandé de prendre en charge les compensations à verser pour ces déplacements de populations. L'État malien, qui n'en a pas les moyens, a demandé à revoir le tracé, afin de diminuer les compensations à verser. Le projet initial a donc été revu afin de diminuer le nombre de villages et de parcelles touchés; le coût prévisionnel des indemnisations a ainsi été ramené à 178000 euros somme qui n'a pas été intégralement versée.

\subsubsection{L'impact du projet sur les ressources en eau disponibles}

Leva payer auli-ibye précise les montants de la redevance que le projet Malbya devra payer au gestionnaire du périmètre pour l'accès à l'eau. Elle est fixée à 4,50 euros/ ha pour les superficies irriguées par aspersion, et 100 euros/ha/an pour les superficies irriguées par gravité, de façon à inciter aux économies d'eau. Cette redevance, pour l'irrigation gravitaire, correspond à $50 \%$ du montant facturé aux paysans, la différence étant censée correspondre à l'investissement réalisé. Cependant les responsables du projet ont négocié pour ne pas payer du tout cette redevance, qu'ils considèrent comme trop élevée étant donné l'effort d'investissement réalisé. Selon Hertzog et al. (2011), il ne s'agit donc plus là seulement d'accaparement foncier, mais également d'accaparement de la ressource en eau.

\subsection{La lente montée de la contestation}

\subsubsection{Les réactions locales}

23 Les nombreux impacts sur leurs moyens d'existence ont engendré frustration et colère chez les paysans (encadré 1 ).

Développement durable et territoires, Vol. 3, n 3 | Décembre 2012 
«Quand les Chinois sont venus creuser le canal pour les Libyens, on ne savait pas ce qu'ils allaient faire. Ils sont venus avec plein d'engins, on avait peur. Les enfants ne sortaient plus du village, car au début des travaux, il y a eu plusieurs morts ». Une femme de Boky Wéré, juillet 2010.

« Pour leur canal et leur grand projet, ils ont détruit des champs et des lieux de passage pour les animaux. Ils ont construit tout un village pour les travailleurs chinois, à la place de nos champs. Ils ont l'eau et l'électricité là-bas, ils ne veulent même pas que nos femme aillent chercher deux bassines d'eau ». Un agriculteur de Boky Wéré, juillet 2010.

« Ma femme doit aujourd'hui faire plus de $10 \mathrm{~km}$ pour traverser le canal et aller chercher du bois, alors qu'avant elle en faisait moins de 2. On ne peut pas non plus utiliser l'eau du canal où les Libyens font leurs tests. On ne peut rien dire car les Libyens sont forts au Mali et amènent beaucoup d'argent ». Un agriculteur de Seriya, août 2010

"C'est seulement après deux ans qu'on a pu avoir de nouvelles maisons et que mon frère a touché un peu d'argent pour le jardin. Mon cousin, lui, n'a rien eu, car pour eux le mil, ça ne vaut rien. Ça a pris beaucoup de temps, ce n'est pas facile de savoir ce que les Libyens vont faire ici, il faut attendre » Un « vieux » de Kolongotomo, juin 2010.

Enquêtes paysannes, 2010.

Ces « sentiments » peinent pourtant à se transformer en réaction collective organisée, et encore plus à se traduire par une action collective d'envergure capable d'influencer le cours du projet. Il est difficile pour les agriculteurs de comprendre exactement ce qui se passe : ils ne disposent pas des informations nécessaires pour pouvoir argumenter contre la mise en œuvre du projet. Et comme le dit un paysan de Kolongotomo, il est difficile de lutter contre un « ennemi » qui n'est pas sur place et qui n'a pas de visage, puisque les employés locaux sont sans pouvoir et sans information.

Cette absence d'information, la lenteur des procédures, les pressions liées aux enjeux politiques du projet, finissent par les décourager, voire par «convaincre » les leaders locaux de la contestation. Le président du syndicat des paysans de la zone a été mis en prison pour trouble à l'ordre public, après avoir manifesté son mécontentement suite à l'expulsion de plusieurs familles paysannes.

Un autre paysan avait monté en 2008 une association pour se plaindre des expulsions non indemnisées. Rencontré en 2010, il nous explique que les Libyens ont respecté leur accord avec le Mali et que les paysans savaient qu'ils allaient s'installer; il met en avant la bonne entente entre le Mali et la Libye et laisse entrevoir les bienfaits du projet: «Le Mali a indemnisé ses paysans, et grâce aux efforts du président, les Libyens ont construit un grand canal et une route pour assurer le développement de la région ». Ce revirement et ce discours "adapté " traduisent les tractations, politiques et financières, visant à atténuer la vigueur des réactions locales. Pourtant, les indemnisations, sont très éloignées des engagements : c'est seulement après deux ans de négociations, et la mobilisation des communes rurales, des sociétés civiles, et des représentants locaux de l'État, que les paysans touchés par le projet ont pu recevoir des indemnités. La somme totale versée s'élève à 10000 euros, soit moins de $6 \%$ de la somme prévue (178000 euros), qui s'appuyait, elle-même, sur une estimation basse des impacts.

Face aux faibles effets de leurs revendications, de nouvelles stratégies apparaissent. Reprenant le discours de l'État sur l'investissement privé, des communes rurales et des groupes de paysans revendiquent le droit de devenir de petits investisseurs et d'accéder à 
des terres aménageables. Individuellement ou regroupés en collectifs, des paysans déposent donc des demandes de bail emphytéotique pour des terres encore gérées traditionnellement par les instances villageoises, qu'elles aient été conservées en forêts ou mises en valeur par l'agriculture pluviale ou le pâturage. Dans l'ensemble, les actions locales restent de faible ampleur (revendication de quelques dizaines de paysans) et ont souvent peu d'impact. Elles commencent cependant à pouvoir compter sur des relais nationaux et internationaux.

\subsubsection{Les actions de la société civile nationale}

Le projet Malibya n'est pas le seul projet d'investissement à grande échelle au Mali. Entre 2000 et 2010, près de 870000 ha $^{8}$ ont été provisoirement attribués à travers des baux à des investisseurs privés ou publics, étrangers ou nationaux. Les responsables agricoles tentent de mobiliser les paysans concernés. À l'échelle nationale, les plus actifs sont le Syndicat des exploitants agricoles de la zone office du Niger (Sexagon) et la Coordination nationale des organisations paysannes (CNOP). Leurs actions sont plus rapidement relayées par les médias, tous basés à Bamako, que les mobilisations strictement locales. La CNOP, en particulier, est devenue un acteur majeur au Mali. Elle regroupe l'ensemble des associations et fédérations paysannes. Elle a ainsi participé à l'élaboration de la loi d'orientation agricole malienne, en 2006, à travers un mémorandum paysan. Malgré son influence grandissante, et bien qu'elle soit devenue un interlocuteur crédible et écouté du ministère de l'Agriculture et des bailleurs de fonds, la portée concrète de son action demeure, à ce jour, limitée.

En juillet 2010, à son initiative, un forum sur les accaparements fonciers s'est tenu à Sélingué, au sud du pays. Des représentants de nombreux pays touchés par le phénomène étaient présents (Congo, RDC, Kenya, Ethiopie, Tanzanie, Tchad, Zambie, Afrique du Sud, Bénin...). Ils ont discuté des directives non-contraignantes FAO/Banque mondiale (FAO et al., 2010) sur les investissements agricoles et des droits des populations locales. Après quatre jours de débats en présence de représentants des organisations internationales (FIDA) et des médias sous-régionaux, ils ont rédigé un rapport exposant leurs positions et leurs revendications.

Forte du succès de ce forum, la CNOP a pu "dynamiser » le syndicat des paysans de la zone ON, appeler les agriculteurs à manifester leur mécontentement face aux accaparements fonciers et organiser une marche de contestation et un forum « local » sur les accaparements fonciers dans la zone Office du Niger elle-même. 


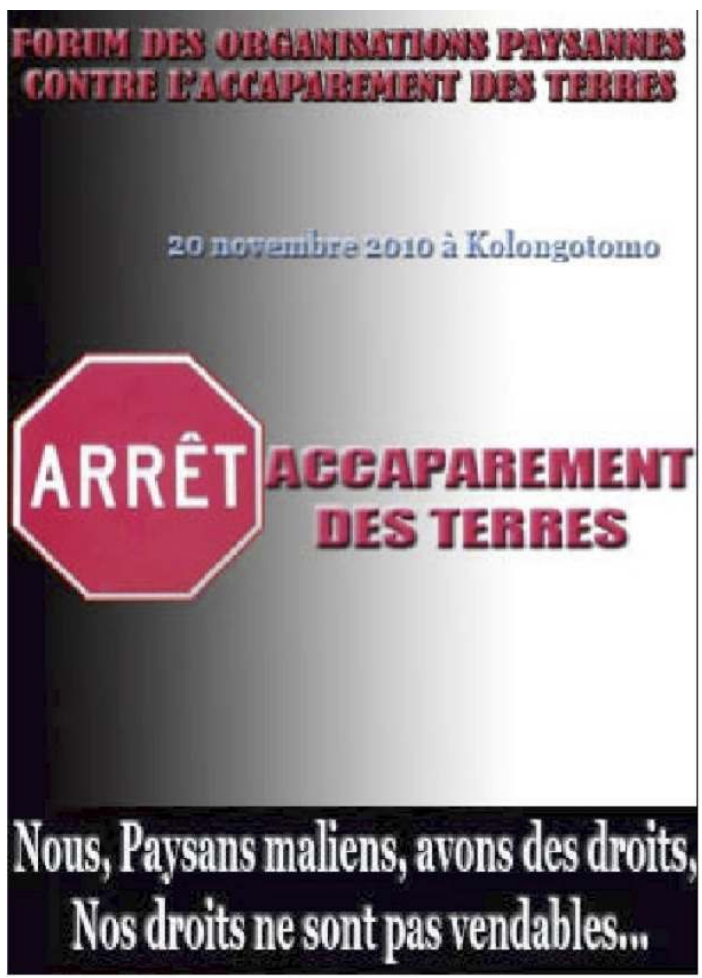
international sur l'accaparement des terres, à Sélingué, en novembre 2011. 250 paysans et acteurs de la société civile, provenant de 30 pays, y ont participé. Un rapport sur l'accaparement des terres, en particulier à l'office du Niger, a été rendu public. Il dénonce plusieurs projets, comme celui de Malibya ou celui des Moulins modernes du Mali ${ }^{10}$. Les paysans y détaillent les expropriations et les violences subies par certains suite à l'arrivée des investisseurs. Malgré la formation d'une «Convergence malienne» contre les accaparements de terres regroupant les principaux acteurs paysans et des organisations plus citadines comme la Ligue des droits de l'homme, les impacts sur le terrain restent limités.

33 La position de la presse nationale malienne est nuancée. Fin 2011, le projet Malibya est décrit par certains journaux et la radio-télévision d'État comme porteur d'un nouveau modèle de développement productif, comme source innovante d'accès aux capitaux et comme un symbole de la coopération interafricaine. D’autres médias sont plus critiques et reprennent les communiqués des organisations paysannes ${ }^{11}$. 


\subsubsection{L'appui des ONG internationales} libyens de 2011 et maliens de 2012. Le canal d'irrigation a été réalisé et mis en eau, les terres ont été bornées... mais aucun aménagement n'a été réalisé. Des expérimentations ont été menées sur 7 ha, en irrigation gravitaire, sur des terres anciennement aménagées de la zone Office du Niger. Les ingénieurs agronomes maliens travaillant pour le projet l'ont quitté en janvier 2011. En novembre 2011, suite aux évènements en Libye, le directeur du projet a quitté le Mali, et les bureaux du projet à Bamako sont fermés. Le nouveau gouvernement libyen privilégie pour l'instant les investissements en Libye. traversées. En fait, de façon plus générale, la réalité de la mise en valeur par les nouveaux projets est souvent bien éloignée des objectifs proclamés. Au-delà des effets d'annonce, de nombreux projets d'investissements ne voient pas le jour. Manque de capitaux disponibles ou absence de maîtrise technique sont habituels. Les promesses productivistes apparaissent ainsi plus comme un argument de "vente » que comme un objectif réel.

Loin des discours sur la croissance et le développement, la constitution de vastes réserves foncières semble alors être l'objectif principal de certains acteurs. L'État affecte des terres à des investisseurs ou à des intermédiaires qui ne respectent pas leurs promesses de mise en valeur et qui acceptent toutes sortes d'arrangements. Ces pratiques sont acceptées car la constitution de réserves foncières sans disposer des capitaux nécessaires à leur mise en valeur, offre, paradoxalement, des opportunités aux paysans ayant besoin de terres. Pour les investisseurs, une des manières de justifier leurs baux, de rentabiliser leurs 
investissements et de payer la redevance en eau, est de sous-louer la terre à des paysans. Certes, c'est d'abord une pratique des investisseurs maliens. Toutefois le projet chinois «COVEC » l'a aussi utilisé. La pratique est illégale et... est acceptée par tous. Les investisseurs préservent leurs possibilités futures de mise en valeur (terres et droits d'eau sont même transmissibles à leur ayants-droits). Les paysans se voient offrir des accès à la terre, certes précaires, mais qu'ils ne pouvaient obtenir dans le cadre des attributions par l'office du Niger. L'État gère ainsi une situation qui pourrait être difficile du point de vue politique : les terres sont utilisées et la contestation diminue.

À court terme, la solution satisfait l'ensemble des acteurs. Qu'en est-il à plus long terme ? De nombreux conflits concernant les sous-locations aboutissent au tribunal d'instance. Peu sont réglés, puisqu'ils concernent une pratique en elle-même illégale. Peut-on croire que l'absence de régulation soit positive? Ne risque-t-on pas, au fur et à mesure de la mise en valeur des terres, de créer des tensions du même type que celles existant dans les terroirs non aménagés : superposition de droits, conflits de légitimité ? Cela serait peu propice à l'investissement et à la mise en valeur.

D'ailleurs, la mobilisation des différents acteurs a contraint l'office du Niger à réviser les attributions et à appliquer, en partie, la réglementation. Ainsi, près de 220000 ha attribués provisoirement à des investisseurs ont fait l'objet de résiliations en 2011. Par ces annulations foncières, l'État cherche à convaincre de la mise en œuvre responsable des choix politiques visant à installer des investisseurs pour développer l'agriculture, en éliminant les cas les plus aberrants. Ces résiliations concernent en majorité (près de $63 \%$ ) des projets de petite taille (de 10 à 50 ha), portés par des petits investisseurs maliens, pour l'essentiel déjà paysans en zone $\mathrm{ON}$. Les grandes conventions, comme celle de Malibya restent, elles, toujours valides, même quand les engagements pris ne sont pas respectés.

\subsection{Les enseignements du projet Malibya}

Quatre principaux éléments peuvent être retenus de l'analyse de ce projet :

- les réactions des agriculteurs et des sociétés civiles peinent à s'organiser, elles n'ont qu'un faible impact local, même si leur impact international peut être plus important grâce au relais des grandes ONG ;

- les rapports de force sont défavorables aux sociétés civiles, face à des alliances complexes entre bailleurs, État, et promoteurs de projet ;

- obtenir l'information est difficile, difficulté renforcée par les réactions de crispation de l'État face aux réactions, qui conduisent à encore plus d'opacité ;

- la faiblesse de l'État, de ses services, de ses normes, fait que ses lois ne sont pas respectées, ni par les investisseurs, ni même par une partie des hommes politiques, qui passent des accords directs avec ces investisseurs.

Au-delà de ces éléments, il faut constater la force du discours de la modernité et l'opinion que la paysannerie ne peut être capable de porter le progrès économique. C'est peut-être le point essentiel. L'agriculture familiale est efficace: les paysans maliens fournissent aujourd'hui l'essentiel des céréales consommées au Mali, y compris pour le riz, base de l'alimentation dans les grandes villes. Mais ni l'agriculture familiale africaine, ni les États n'ont les moyens de financer les investissements nécessaires à la grande irrigation. Et il est probable que la modernisation des agricultures familiales se traduira par des concentrations de terres, à l'image de ce qui s'est produit en Europe et aux USA... 

stratégies des acteurs des "sociétés civiles", qui sont face à un choix: lutter frontalement contre ces projets d'investissements et promouvoir le seul développement de l'agriculture familiale, ou composer avec ces nouveaux acteurs, pour imaginer de nouveaux modèles de développement, permettant de résoudre la contradiction principale qui est que, malgré leurs succès techniques, ni les paysans ni l'État n'ont les moyens d'investir.

\section{Quelles perspectives d'action pour les sociétés civiles?}

\subsection{Composer ou s'opposer?}

lutte frontale peut apparaître comme la solution la plus appropriée. Cette stratégie a été couronnée de succès dans le cas du premier et du plus emblématique des grands cas d'appropriation foncière, celui de Daewoo à Madagascar. En 2009, l'annonce de ce projet sud-coréen portant sur plus d'un million d'hectares a fait la une de la presse internationale. Le terme d'accaparement foncier a été largement utilisé (Brondeau, 2011). L'impact médiatique s'explique, notamment, par le soulèvement de plusieurs milliers de personnes qui a conduit au renversement du gouvernement en place. L'exemple est cependant peu représentatif. L'annonce du projet a été faite au moment où une lutte politique aigüe divisait le pays et opposait deux partis qui se disputaient le pouvoir. Ce n'est pas le cas dans tous les pays, où la faiblesse de l'État se combine souvent avec le contrôle très strict des politiques et du Président lui-même sur tous les leviers de commande. L'ampleur et l'irréalisme du projet étaient tels que les arguments économiques ne pouvaient pas convaincre. La transparence a été relativement grande et a nui aux promoteurs.

Dans la plupart des projets d'investissement, les possibilités de contestation sont réduites, du simple fait qu'au stade de la négociation avec l'État hôte il est très souvent difficile de connaître les composantes d'un projet, et donc de le contester. Nous l'avons vu pour le Mali, les populations sont informées quand tout est déjà décidé, et il est alors presque impossible de stopper des projets porteurs de plusieurs millions d'euros, en dépit de leurs conséquences sociales.

Lutter contre l'opacité (veil of secrecy, dans le Rapport de la Banque mondiale de 2010) pourrait donc être une première priorité pour tous ceux qui veulent investir ce champ d'action. La Banque mondiale elle-même s'estime victime de cette opacité, puisque, face aux réticences des États et des firmes à fournir leurs chiffres, elle a dû, pour mener à bien son étude, s'appuyer sur les données diffusées par l'ONG GRAIN, qu'elle savait pourtant très hostile à ces investissements. Demander le respect des lois et des réglementations en vigueur pourrait être un second axe d'action. Les lois sur les études d'impact environnemental et social des investissements doivent être appliquées.

Un troisième axe serait de demander une meilleure intégration des populations locales dans les processus d'étude et de réalisation des projets, pour garantir de réelles retombées économiques, éviter la constitution de rentes foncières et se prémunir de conséquences sociales et écologiques négatives. 

la concentration des terres. Les paysans pauvres, poussés à apporter leur terre en garantie d'un crédit, ou à la vendre en cas de besoins, deviennent plus vulnérables. Le marché foncier risque de faciliter la réaffectation des terres en faveur des acteurs les plus performants - ou les plus riches - (Nyamu Musembi, 2008), éliminant les ménages ruraux pour lesquels l'agriculture n'est pas assez rentable, ou qui sont trop pauvres pour résister à l'attrait de sommes d'argent De nombreux travaux montrent la relation négative entre concentration foncière et pauvreté. La réduction des inégalités de répartition foncière fait baisser la pauvreté bien plus rapidement que la croissance agricole (El-Ghonemy, 2003).

Les défenseurs des agricultures paysannes objectent que la reconnaissance des droits existants exclut toute possibilité de réforme agraire, réforme qui viserait à fournir un accès foncier aux sans terre, ou à élargir le foncier accessible aux paysans pauvres qui disposent de parcelles de plus en plus petites ${ }^{14}$, trop petites pour pouvoir se sortir de leur pauvreté. Ces différentes propositions se retrouvent largement dans les codes de bonne conduite, nombreux à défaut d'être appliqués, qui tentent de «moraliser» les investissements. Le plus médiatisé et le plus évoqué a été proposé conjointement par la FAO, le FIDA, la CNUCED et la Banque mondiale, en janvier 2010 (FAO et al., 2010). Ces principes sont présentés dans l'encadré 2 .

\section{ENCADRÉ 2 : LES SEPT PRINCIPES DE L'AgRO-INVESTISSEMENT RESPONSABLE}

Principles for Responsible Agricultural Investment that Respects Rights, Livelihoods and
Resources
Principle 1 : Existing rights to land and associated natural resources are recognized and
respected.
Principle 2 : Investments do not jeopardize food security but rather strengthen it.
Principle 3 : Processes for accessing land and other resources and then making associated
investments are transparent, monitored, and ensure accountability by all stakeholders,
within a proper business, legal, and regulatory environment.
Principle 4: All those materially affected are consulted and agreements from




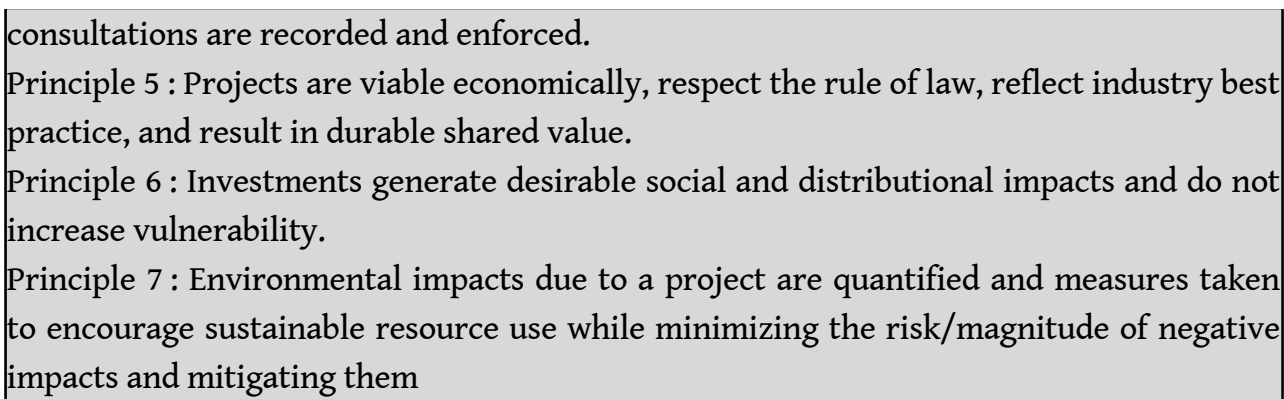

Il existe donc une prise de conscience internationale liée aux risques d'accaparement. Les déclarations et les textes devant régir le développement de ces projets restent des outils de gouvernance internationale, des productions institutionnelles qui ne permettent pas de contrôler le phénomène. Ils visent à garantir « un investissement agricole responsable qui respecte les droits, les moyens d'existence et les ressources ». Leur inefficacité provient du fait qu'ils n'ont aucun caractère contraignant. Leur application éventuelle relève d'une démarche volontaire des investisseurs. En pratique, ces principes sont rarement appliqués (Borras et Franco, 2010). Malgré la multiplication des traités internationaux du type des directives volontaires (Déclarations des Nations unies, du FMI...), on observe une augmentation sans précédent des cas de violations des droits des populations autochtones (Sawyer et Gomez, 2008).

Les difficultés d'application de ces textes proviennent également de la «mauvaise gouvernance ", qui relève d'abord de la défaillance d'États faibles, et mal préparés pour faire face à cette vague d'investissements fonciers. À défaut d'obtenir des firmes qu'elles investissent de manière responsable, on pourrait demander aux États d'accueil de l'exiger dans les conventions qu'ils passent. Cela supposerait de renforcer leurs capacités juridiques et leurs ressources propres, de façon à ce qu'ils puissent mieux résister à l'attrait de financements indélicats.

Plus de 120 ONG, nationales et internationales, ont signé en avril 2010 une déclaration d'opposition aux « sept principes " pour un investissement foncier responsable, précisant $q^{\prime} u^{\prime}$ aucun principe au monde ne peut justifier l'accaparement de terres ${ }^{15}$. Cette stratégie d'opposition, a cependant, sauf exceptions, beaucoup de mal à se concrétiser car les alternatives et les moyens de résister manquent aux acteurs locaux.

\subsection{Peut-on imaginer de changer complètement de cadre de référence?}

Plutôt que de placer l'agriculture familiale dans une position défensive, face à l'agriculture d'entreprise, la question est peut-être de donner à l'agriculture familiale une place pleine et entière, bien définie dans les politiques publiques (Tonneau et al., 2005), correspondant à ses capacités et prenant en compte ses limites et ses besoins. L'expérience du Brésil est à cet égard intéressante (Andion, 2006). Deux voies y ont été explorées depuis une vingtaine d'années : celle de la complémentarité économique entre agricultures familiales et exploitations agro-industrielles, mais aussi celle d'une plus grande autonomie des exploitations familiales, en les spécialisant sur le créneau de la sécurité alimentaire des campagnes et des petites villes. 
exploitations agro-industrielles. Cette agriculture contractuelle est réputée permettre aux exploitations familiales de mieux s'intégrer aux marchés mondiaux, de participer aux nouveaux marchés de produits à haute valeur ajoutée, d'améliorer la qualité de leurs produits, de stabiliser leurs revenus, et de profiter de transferts de techniques à même d'accroitre leur productivité. Le manque de régulation et les rapports déséquilibrés entre acteurs remettent en cause la notion même de contrat (Prowse, 2008). La contractualisation peut, vite, n'être qu'un moyen de capter la valeur ajoutée produite par des agriculteurs familiaux dont les revenus sont inférieurs à ceux d'un salarié.

Une exploitation capitaliste, pour être rentable, a besoin d'un accès très peu coûteux au foncier, et cela motive les mécanismes de l'accaparement foncier. Elle doit également diminuer ses coûts de main-d'œuvre, soit en supprimant le facteur travail des processus de production, par la mécanisation, soit en le sous-traitant à des agriculteurs familiaux qui ne comptabilisent pas leur temps de travail. Il peut ainsi être plus rentable pour une firme privée de passer des contrats avec des exploitations familiales ou de petits collectifs, pour ses approvisionnements en matières premières ou pour accroittre ses volumes commercialisés.

Les expériences d'agriculture contractuelle n'ont été jusqu'à maintenant que peu couronnées de succès. Peut-être que, comme le dénoncent certaines ONG, elles ne sont que des alibis. C'est là que l'expérience brésilienne peut être mobilisée. L'État a reconnu différents projets pour l'agriculture familiale: un projet de producteurs ruraux, insérés dans l'économie internationale, au travers de contrats ; un projet paysan d'autosuffisance alimentaire, projet accompagné de politiques sociales et d'infrastructures (Tonneau et al., 2010). Des perspectives sont ouvertes et les « sociétés civiles » s'engagent en partenariat, parfois difficile avec l'État, dans la mise en œuvre de propositions alternatives (Schneider et Niederle, 2010). Il ne s'agit pas d'idéaliser la situation brésilienne (Ondetti, 2008). La force de la «modernité » reste déterminante et les politiques d'appuis aux agricultures familiales sont souvent présentées comme transitoires, dans l'attente du développement du salariat, dans l'agriculture et les autres secteurs.

61 En Afrique, sauf de rares exceptions, ni les États, ni les bailleurs de fonds, ni les représentants des agriculteurs familiaux n'ont réellement investi ce champ pour inventer des systèmes qui soient réellement gagnant-gagnant, ou pour faire en sorte que la plusvalue soit partagée entre les deux parties d'une manière "équitable », donc durable ( durable shared value, dans le vocabulaire de la Banque mondiale).

Ce manque d'expérimentations sociales est probablement lié à un manque de confiance généralisé. On revient ici à la question de la nécessité de rapports de force plus équilibrés et au besoin de régulations acceptées par tous ou imposées à tous par l'État. L'expérience du Brésil n'a pu se faire que grâce à une volonté forte de l'État d'investir dans le soutien financier aux agriculteurs familiaux, soutien qui fait l'objet d'un consensus, qui se traduit au plan politique par l'existence d'un ministère spécifique, à même de mobiliser les moyens d'un État fédéral puissant, qui dirige une des économies les plus dynamiques du monde actuel.

Ce soutien concerne en fait toutes les formes d'agricultures familiales du Brésil, ce qui permet d'explorer leur rôle possible dans la sécurité alimentaire ou dans une agriculture, impactant moins l'environnement, car basée sur les principes reconnus (Griffon, 2006) de l'agro-écologie (Altieri, 2002) et sur une intensification en travail. On s'éloigne ainsi d'une 
logique dominante dans les modèles agro-industriels, où se pose la question des externalités négatives (Dufumier et Lallau, 2010).

Investir dans la construction de nouvelles propositions pour l'agriculture familiale et les relations de cette dernière avec les entreprises semble une nécessité dans tous les pays concernés par ce type de problème. Cela implique de créer un climat de confiance minimum. Cela dépend d'une «bonne gouvernance» qui, par ailleurs, est identifiée comme "facteur déterminant » pour le développement. L'exemple brésilien n'est pas facile à extrapoler. Des pays dont l'économie est en voie de décollage, comme le Maroc, qui dispose d'une structure étatique solide et stable, ont cependant mis en place des dispositifs similaires: le Plan Maroc vert s'appuie ainsi sur deux piliers, l'agriculture moderne privée et l'agriculture traditionnelle et vivrière; il promeut les partenariats public-privé et "l'agrégation », un partenariat entre petits exploitants regroupés autour d'un agro-industriel ${ }^{17}$. Dans le cas de pays sub-sahariens fragiles et pauvres comme le Mali, ce type d'expérimentation n'est pas aisé à envisager sans de sérieux appuis financiers et institutionnels extérieurs.

\section{Conclusion}

L'analyse du projet Malibya au Mali nous a permis de mettre en évidence les impacts immédiats de l'accaparement foncier sur les exploitations familiales. À plus long terme, les difficultés techniques et financières de mise en œuvre des grands projets d'investissement contribuent à la constitution d'une rente foncière, associée, dans le cas des périmètres irrigués, à une rente hydrique. Nous avons pu constater la faiblesse des États; le déficit de gouvernance ne favorise pas le climat nécessaire à une coopération entre firmes privées et agriculteurs familiaux, qui pourrait permettre d'améliorer la durabilité des projets proposés, et qui pourrait peut-être permettre de concilier impératifs économiques, sociaux et environnementaux.

La question fondamentale est celle de la place future des agricultures familiales, qui existent et produisent, mais qui doivent évoluer pour subsister dans un environnement où l'accès aux marchés est indispensable, et où l'agriculture - surtout irriguée - nécessite des investissements coûteux. En nous appuyant sur l'expérience brésilienne, nous pensons que des expérimentations sont nécessaires pour explorer, à la fois les complémentarités possibles entre agricultures familiales et entreprises et les possibilités de développement d'une agriculture familiale autonome. Les conditions de réalisation de telles expériences sont cependant à étudier au cas par cas. Dans le cas des pays les plus fragiles au plan économique et au plan institutionnel, un accompagnement extérieur semble indispensable tant les rapports de force sont déséquilibrés.

Dans cette perspective, l'action de la société civile pourrait s'organiser autour de deux axes: 1) La lutte pour le respect des lois et des réglementations existantes dans le cas d'installation d'entreprises (indemnisation, compensation sociale par des infrastructures sociales). 2) L'expérimentation autour des complémentarités agriculture familiale et entreprises d'une part, et sur l'autonomie des agricultures familiales, d'autre part. Cela signifie travailler avec l'ensemble des types d'agriculture familiale (et pas seulement les producteurs familiaux les plus capables de s'intégrer aux marchés internationaux), expérimenter de nouvelles solutions, sans exclusive, et soutenir financièrement et techniquement les agricultures familiales. 
68 originales, refusant, ou tout au moins gérant, la fatalité de la diminution de la population agricole. De telles perspectives sont-elles utopiques en Afrique? Probablement encore dans beaucoup de pays, sauf à renforcer et à faire réellement vivre des dispositifs favorisant une meilleure gouvernance, et permettant un renforcement des compétences des citoyens et des institutions de l'État.

\section{BIBLIOGRAPHIE}

Adamczewski A., Burnod P., Jamin JY., Tonneau JP., 2010, “Investments in irrigable land for largescale agricultural production in Mali "International Conference Africa for Sale : Analysing and Theorizing foreign Land Claims and Acquisitions, Groningen, Netherlands. 28-29 October 2010, $18 \mathrm{p}$.

AGTER, 2010, « Appropriation et concentration de droits fonciers à grande échelle. Le cas du Mali », janvier 2010 : http://farmlandgrab.org/10462

Alternatives économiques, 2009, Afrique main-basse sur la terre. Alternatives Économiques $\mathrm{n}^{\circ} 276$ janvier 2009. http://www.alternatives-economiques.fr/afrique--main-basse-sur-laterre_fr_art_809_41502.html

Altieri M., 2002, Agroecological principles for sustainable agriculture. Agroecological innovations increasing food production with partipatory development, London,Uphoff N. editor, Earthscan.

Andion C., 2006, « Développement territorial durable en milieu rural, gouvernance et rôle des organisations non gouvernementales : l'État de Santa Catarina au Brésil », Mondes en développement 4/2006 ( $\left.\mathrm{n}^{\circ} 136\right)$, p. 85-100. URL : www.cairn.info/revue-mondes-endeveloppement-2006-4-page-85.htm. DOI : 10.3917/med.136.0085.

Anseeuw W., Boche M., Breu T., Giger M., Lay J., Messerli P., Nolte K., 2012, Transnational Land Deals for Agriculture in the Global South. Analytical Report based on the Land Matrix Database. CDE/ CIRAD/GIGA, Bern/Montpellier/Hamburg.

Baxter J., 2010, « Ruée sur les terres africaines », Le Monde Diplomatique, 670 (01/201) p. 18 http:// www.monde diplomatique.fr/2010/01/BAXTER/18713

Borras S., Franco J.C., 2010, "From threat to opportunity ? Problems with the idea of a 'code of conduct' for land-grabbing", Yale Human Rights and Development Law Journal, 13, 1, p. 507-523

Brondeau F., 2011, « L'agro-business à l'assaut des terres irriguées. Quelles perspectives de développement dans les systèmes irrigués de l'office du Niger? »Cahiers Agricultures 20, 1-2, p. 136-142. doi : 10.1684/agr.2011. 0472

Burnod P., Papazian H., Adamczewski A., Bosc P.M., Tonneau J.P., Jamin J.Y., 2011, « Régulations des investissements agricoles à grande échelle. Études de Madagascar et du Mali », Afrique contemporaine, 2011/1 n²37, p. 111-129. DOI : 10.3917/afco.237.0111

Chouquer G., 2011, « Le nouveau commerce triangulaire ou les analogies du foncier contemporain », Études rurales, 187, p. 95-130. 
Clavé M., 2010, « Les cessions d'actifs agricoles à des investisseurs étrangers dans les pays en développement. Éléments de diagnostic et pistes de recommandations », Juin, Centre d'analyse stratégique, Rapports et Documents.

Cicodev Africa, 2011, Accaparement de terres en Afrique de l'Ouest. Exporter ou nourrir les populations. Impacts sur les consommateurs ruraux, 25 p. http://www.hubrural.org/IMG/pdf/ accaparement_des_terres_rapport_diokoul_1sur2.pdf

Cotula L., Vermeulen S., Leonard R., Keeley J., 2009, “Land grab or development opportunity? Agricultural investment and international land deals in Africa", FAO, IIED and IFAD.

Delcourt L., 2010, «L'avenir des agricultures paysannes face aux nouvelles pressions sur la terre ", CETRI, www.cetri.be/spip.php ?article1806.

De Schutter O., 2009, "Large-scale land acquisitions and leases : A set of core principles and measures to address the human rights challenge", UNO, 11 June.

De Schutter O., 2010, Accès à la terre et droit à l'alimentation, Rapport devant la 65ième session de l'Assemblée générale des Nations Unies (troisième commission), 11 août.

Deininger K., Binswanger H., 1999, “The evolution of the World Bank's land policy : principles, experiences and future challenges", World Bank Research Observer, 14 (2).

Dufumier M., 2012, Famines au Sud, malbouffe au Nord. Comment le bio peut nous sauver, Paris, Nil Editions.

Dufumier M., Lallau B., 2010, « Agriculture et développement durable », in : B. Zuindeau (dir.), Développement durable et territoire, Villeneuve d'Ascq, Presses Universitaires du Septentrion.

El-Ghonemy M.R., 2003, Land reform development challenges of 1963 - 2003 continue into the twenty-first century, in FAO, Réforme agraire, colonisation et coopératives agricoles, 2003/2. (http:// www.fao.org/docrep/006/j0415t/j0415t00.htm\#Contents)

FAO, IFAD, UNCTAD, World Bank Group, 2010, Principles for Responsible Agricultural Investment that Respects Rights, Livelihoods and Resources, Discussion note, January 25.

GRAIN, 2008, "Seized! The 2008 Land Grabbers for Food and Financial Security", October.

GRAIN, 2009, «L'accaparement des terres de rizières met en péril la souveraineté alimentaire de l'Afrique », Against the grain, janvier.

Griffon M., 2006, Nourrir la planète, Paris, Ed. Odile Jacob.

Hertzog T., Adamczewski A., Molle F., Poussin J.C., Jamin J.Y., 2012, “" Ostrich-like » Strategies in Sahelian Sands ? Land and Water Grabbing in the Office du Niger, Mali”, Water Alternatives, 5 (2), p. 304-321.

IFAD, 2010, Rural Poverty Report 2011. New realities, new challenges : new opportunities for tomorrow's generation, IFAD, December, Rome.

Khilnani S., 2001, «La société civile, une résurgence », Critique internationale, 1/2001, nº 10, p. 38-50. URL : www.cairn.info/revue-critique-internationale-2001-1-page-38.htm. DOI : 10.3917/ crii.010.0038.

Lafargue F., 2005, « La Chine, une puissance africaine », Perspectives chinoises, 90. http:// perspectiveschinoises.revues.org/900

Lautier B., 2002, « Pourquoi faut-il aider les pauvres? Une étude critique du discours de la Banque mondiale sur la pauvreté », Tiers-Monde, 43, n²169, p. 137-145. 
Le Quorum, 2011, « Chronique de la consommation, de la terre à la table », Le quorum, Décembre 2011. http://www.lequorum.com/analyses/2012-1159-chronique-de-la-consommation-de-laterre-a-la-table

Martínez-Torres M., Rosset P., 2010, "La Vía Campesina : the birth and evolution of a transnational social movement", Journal of Peasant Studies, 37, p. 149-175.

Mazoyer M., Roudart L., Mayaki I.A., 2008, « Rapport sur le développement dans le monde, Banque mondiale, L'agriculture au service du développement. Résumé et commentaires", Mondes en développement, 2008/3, n 143, p. 117-136.

Nyamu Musembi C., 2008, "De Soto and land relations in Africa : breathing life into dead theories about property rights”, in: Saturnino M., Borras Jr. (eds.), Routledge, .Market-Led Agrarian Reform : Critical Perspectives on Neoliberal Land Policies and the Rural Poor.

Olivier Martin J., 2011, « Louis Dreyfus n'entrera pas en Bourse », Le Figaro, 7/12/2011. http:// www.lefigaro.fr/societes/2011/12/07/04015-20111207ARTFIG00700-louis-dreyfus-n-entrera-pasen-bourse.php

Ondetti G., 2008, Land, protest, and politics : the Landless Movement and the struggle for agrarian reform in Brazil, University Park, Pennsylvania State University Press.

Otayek R., 2002, «Vu d'Afrique ? Société civile et démocratie », Revue internationale de politique comparée 2/2002 (Vol. 9), p. 193-212.URL : www.cairn.info/revue-internationale-de-politiquecomparee-2002-2-page-193.htm. DOI : 10.3917/ripc.092.0193.

Prowse M., 2008, « Faire fonctionner l'agriculture contractuelle », Capacity.org, n 34, Août.

Rangeon F., 1986, « Société civile : Histoire d'un mot ", in Jacques Chevallier et al., La société civile, Paris, PUF, p. 9-32.

Rochegude A., 2011, « La terre objet et condition des investissements agricoles. Quels droits fonciers pour l'Afrique?», Afrique contemporaine, $\mathrm{n}^{\circ} 237$.

Rowe G., L. J. Frewer, 2004, “Evaluating public participation exercises : a research agenda", Science, Technology, and Human Values, 29, 4, p. 512-556.

Sawyer S., E. Gomez, 2008, Transnational governmentality and resource extraction : indigenous peoples, multinational corporations, multilateral institutions and the state. Geneva, UNRISD.

Schneider S., Niederle P., 2010, "Resistance strategies and diversification of rural livelihoods : the construction of autonomy among Brazilian family farmers", Journal of Peasant Studies, 37, p. 379-405.

Shepard D., Mittal A., 2009, The Great Land Grab. Rush for World's Farmland Threatens Food Security for the Poor, Oakland Institute.

Teyssier A., Ramarojohn L., Andrianirina Ratsialonana R., 2010, « Des terres pour l'agro-industrie internationale ? Un dilemme pour la politique foncière malgache », Echogéo, 11 Décembre 2009, février 2010.

Tonneau J.P., De Aquino J.R., Teixeira O.A., 2005, “Agriculture policy dilemma : modernisation of family farming and exclusion”, Cahiers Agricultures, 14, 1, p. 30-34. http://www.john-libbey eurotext.fr/fr/revues/agro_biotech/agr/edocs/00/04/0D/68/article.md ?type =text.html Tonneau J.P., Sidersky P., Eloy L., Sabourin E., 2010, « Dynamiques et enjeux des agricultures familiales au Brésil », Géoconfluences (Dossier Le Brésil, ferme du monde ?) : 13 p. [20100323]. http://geoconfluences.ens-lsh.fr/doc/etpays/Bresil/BresilScient7.htm 
Vermeulen S., Cotula L., 2010, "Making the most of agricultural investment : A survey of business models that provide opportunities for smallholders", FAO and IIED.

Von Braun J., Meinzen-Dick R., 2009, “Land Grabbing” by Foreign Investors in Developing Countries : Risks and Opportunities, IFPRI Policy Brief 13, April.

World Bank, 2008, World Development report. Agriculture for Development, Washington DC.

World Bank, 2009, "Large scale acquisition of land rights for agricultural or natural resourcebased use", Concept note, February, 18. http://siteresources.worldbank.org/INTARD/Resources/ sleeping_giant.pdf

World Bank, 2010, "Rising Global Interest in Farmland. Can it Yield Sustainable and Equitable Benefits ?" September, Washington DC.

\section{ANNEXES}

ANNEXE 1 : Appel de Kolongo 20 novembre 2010 http://pubs.iied.org/pdfs/G03056.pdf

\section{NOTES}

1. Le recours aux données d'une ONG illustre les difficultés pour obtenir de l'information auprès des investisseurs et des États, même pour la Banque mondiale.

2. Le portail LandMatrix http://landportal.info/fr/node/10290 et le rapport sur les «Transactions foncières transnationales pour l'agriculture dans l'hémisphère sud " sont issus d'une collaboration mobilisant une quarantaine d'organisations membres de l'ILC (International Land Coalition), dont le CIRAD (Centre de coopération internationale en recherche agronomique pour le développement).

3. RFI (janvier 2009) MFI Hebdo, Politique - diplomatie. Question internationale : opportunité économique ou néo-colonialisme agraire? http://www.rfi.fr/fichiers/MFI/ PolitiqueDiplomatie/2721.asp

4. Le message selon lequel le soutien aux agricultures familiales constitue le plus sûr moyen de réduire la pauvreté rurale est d'ailleurs réaffirmé dans le dernier rapport sur la pauvreté rurale du Fonds international pour le développement agricole (IFAD, 2010).

5. Ainsi, le rapport du Centre d'analyse stratégique (Clavé, 2010) constitue d'abord un plaidoyer pour défendre la légitimité des investissements agricoles français et européens à l'étranger.

6. Lancé dans les années 1930, l'Office du Niger est un haut lieu de la production rizicole malienne, au sein duquel des paysans maliens installés par l'État cultivent 100000 ha irrigués par gravité.

7. Comme le déclare un haut responsable politique malien «les libyens ont demandé, suite à l'étude technique, un débit de $130 \mathrm{~m}^{3} / \mathrm{s}$, mais il n'est pas dit qu'on va le leur donner, ça dépendra des projets et des besoins en eau de l'ensemble de la zone ». Lorsque l'ambassadeur l'appelle à ce sujet, ce responsable l'assure que le gouvernement fera son maximum, afin de préserver les bonnes relations entre les deux pays, sans toutefois trop s'engager officiellement.

8. Pic des attributions en zone Office du Niger (Note officielle Ségou, Mai 2010).

9. Interview d'un responsable de l'office du Niger, Août 2011.

10.

http://www.inter-reseaux.org/IMG/pdf/

Fiche_ECADIM_MALI_version_PDF_6_juillet_2011.pdf 
11. http://www.info-matin.com/index.php/actualite11/2460-convergence-malienne-contre-lesaccaparements-des-terres-lettre-ouverte-au-ministre-de-lagriculture ; http://essor.ml/ actualite/article/lancement-de-la-campagne-agricole

12. http://www.alimenterre.org/ressource/situation-politique-et-accaparement-terres-mali ; http://www.inter-reseaux.org/IMG/pdf/Itw_de_Coulibaly_Lome_avril2012_.pdf

13. Forum social mondial, Dakar 2011. http://fsm2011.org/fr/fsm-2011

14. De Schutter (2010) relève ainsi la chute importante de la superficie moyenne des exploitations en Inde, passée de 2,6 ha à 1,4 ha entre 1960 et 2000, et la chute continue depuis, comme au Bangladesh, en Thaïlande, ou en Afrique orientale et australe.

15. http://www.grain.org/o_files/wb-landgrab-2010-fr.pdf

16. Ce modèle est notamment examiné par une étude de l'IIED (Vermeulen et Cotula, 2010).

17. http://www.ada.gov.ma/Plan_Maroc_Vert/plan-maroc-vert.php

\section{RÉSUMÉS}

Le Mali est, comme d'autres pays d'Afrique, touché par le phénomène des investissements fonciers à grande échelle, qualifiés par certains d'accaparements. Dans la zone irriguée de l'office du Niger, le gouvernement a provisoirement attribué près de 600000 ha à des investisseurs, étrangers et nationaux, publics et privés. Face au manque de régulation par l'État des ces investissements, qu'il a lui-même sollicités, les sociétés civiles maliennes tentent de s'organiser. En s'appuyant sur l'exemple du projet Malibya, l'article étudie les réactions locales à la mise en œuvre d'un projet d'investissement. L'analyse des possibilités d'action qui s'offrent aux populations conduit à poser les questions de la gouvernance d'États faibles, de la place des sociétés civiles, de l'avenir de l'agriculture familiale, et de sa cohabitation avec l'agro-industrie.

Like other African countries, Mali is affected by the phenomenon of foreign investments and land grabbing. In the irrigated area of the Office du Niger, the government provisionally attributed nearly 600000 ha to public and private, foreign and domestic, investors. Given the lack of public regulation of these investments, civil societies try to get themselves organized. Based on the example of the Malibya project, the paper analyses the implementation of an investment project and the local reactions. It then examines the options that are available to people facing these investments. Issues like governance of weak states, civil societies' role, future of family farming and its cohabitation with agribusiness, are discussed.

\section{INDEX}

Mots-clés : accaparements fonciers, agriculture familiale, investisseurs, Mali, sociétés civiles

Keywords : civil society, family farming, investors, land grabbing, Mali 


\section{AUTEURS}

\section{AMANDINE ADAMCZEWSKI}

Amandine Adamczewski est doctorante en Géographie. Son travail de recherche porte sur la gestion foncière des terres en zone Office du Niger au Mali, les dynamiques d'investissements, et les logiques d'acteurs. Cirad UMR G-Eau, TA C-90/15, 73, rue Jean-François Breton, 34398 Montpellier Cedex 5, Tel. +33 (0)4 671664 87, amandine.adamczewski@cirad.fr

\section{JEAN-YVES JAMIN}

Jean-Yves Jamin est agronome (Cirad, UMR G-Eau). Il travaille sur l'évolution des systèmes production dans les périmètres irrigués et sur les relations entre l'eau, l'agriculture et les secteurs non-agricoles. Cirad UMR G-Eau, TA C-90/15, 73, rue Jean-François Breton, 34398 Montpellier Cedex 5, Tel. +33 (0)4 67166485 - jamin@cirad.fr

\section{BENOÎT LALLAU}

Benoît Lallau est maître de conférences à l'Université Lille 1, économiste, membre du CLERSE (UMR CNRS). Ses travaux portent sur les vulnérabilités et la résilience des agriculteurs, en particulier sur différents terrains africains. Il travaille en collaboration avec le LERSA (Laboratoire d'Économie Rurale et de Sécurité Alimentaire de l'Université de Bangui). Université Lille1, Bâtiment SH2, 59655 Villeneuve d'Ascq Cedex, benoit.lallau@univ-lille1.fr

\section{JEAN-PHILIPPE TONNEAU}

Jean-Philippe Tonneau, agronome et géographe, est directeur de l'UMR Tetis. Ses travaux portent sur l'organisation spatiale des territoires, les modèles de développement, les politiques publiques et l'élaboration de plans et de schémas régionaux d'aménagement. Maison de la Télédetection 500 rue Jean François Breton TA C-91 / MTD (Bât. M. Téléd., Bur. 105) -34398 Montpellier Cedex Tél : +33 467548715 - jean-philippe.tonneau@cirad.fr 